\title{
Potencial acaricida do óleo de andiroba Carapa guianensis Aubl. sobre fêmeas adultas ingurgitadas de Anocentor nitens Neumann, 1897 e Rhipicephalus sanguineus Latreille, 1806
}

\author{
[Acaricide potencial of andiroba (Carapa guianensis Aubl.) oil on engorged adult females of Anocentor nitens \\ (Neumann, 1897) and Rhipicephalus sanguineus (Latreille, 1806)]

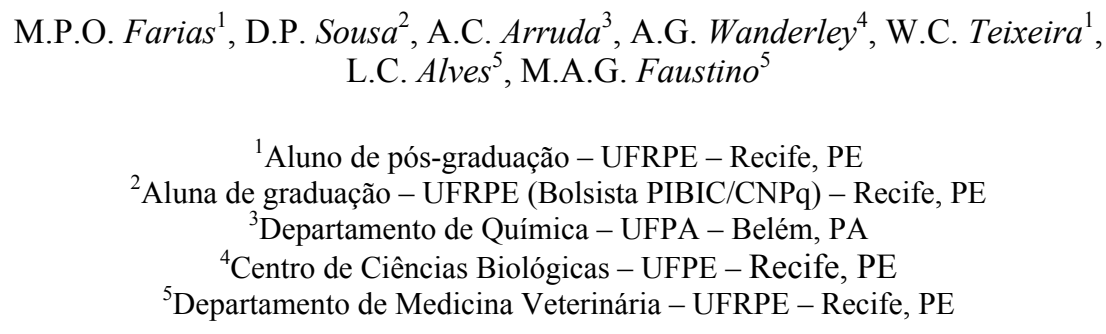

\section{RESUMO}

Avaliou-se o potencial acaricida in vitro do óleo da semente da andiroba (Carapa guianensis) sobre fêmeas ingurgitadas de Anocentor nitens $(\mathrm{n}=210)$ e Rhipicephalus sanguineus $(\mathrm{n}=140)$, coletadas manualmente, respectivamente, de equinos e de cães naturalmente infestados. Para o teste de imersão, empregaram-se cinco diluições do óleo de andiroba, 100\%, 50\%, 30\%, 25\% e 10\%, em água destilada, utilizando-se tween 80 como dispersante. No teste com $A$. nitens, foram usadas três repetições para cada diluição, utilizando-se 10 fêmeas ingurgitadas para cada tratamento. No teste com $R$. sanguineus, usaramse duas repetições, e formaram-se, ainda, dois grupos-controle para cada espécie de ixodídeo, um com água destilada e outro com tween 80 mais água destilada. Após os testes, as fêmeas foram mantidas em laboratório sob temperatura ambiente. Observou-se mortalidade das fêmeas ingurgitadas e redução de postura, neste caso, com ovos inférteis, demonstrando eficácia de $100 \%$ nas duas espécies em todas as diluições testadas. Os dados obtidos evidenciaram a potencialidade do uso do extrato de andiroba contra A. nitens e $R$. sanguineus.

Palavras-chave: carrapato, controle, fitoterápico, andiroba, produtos naturais

\begin{abstract}
In vitro acaricide potential of the oil from andiroba seed (Carapa guianensis) was evaluated on engorged females of Anocentor nitens $(n=210)$ and Rhipicephalus sanguineus $(n=140)$ manually collected, from horses and dogs naturally infested, respectively. Five dilutions, 100\%, 50\%, 30\%, 25\%, and 10\% of andiroba seed oil in deionized water, using tween 80 as dispersant, were employed for the engorged females immersion test. For A. nitens test, three repetitions were made with each dilution, using 10 engorged females for each treatment, and two repetitions for $\mathrm{R}$. sanguineus test. Two control groups were used for each tick species, one with deionized water and another one with tween 80 and deionized water. After the tests, the females were kept in the laboratory under room temperature. Engorged female mortality and oviposition reduction were observed with infertile eggs, showing $100 \%$ of efficacy in the two species in all tested dilutions. The obtained data demonstrated the potential use of andiroba seed extract against $\mathrm{A}$. nitens and $\mathrm{R}$. sanguineus.
\end{abstract}

Keywords: tick, control, phytotherapics, andiroba, natural products

Recebido em 24 de novembro de 2008

Aceito em 18 de junho de 2009

E-mail: marcia_paula529@hotmail.com 


\section{INTRODUÇÃO}

Os carrapatos têm importância econômica por determinarem perdas na produção e no desenvolvimento dos animais, além de serem importantes transmissores de diversos patógenos tanto para o homem como para o animal (Labruna e Pereira, 2001; Monteiro et al., 2003; Soares et al., 2006).

O carrapato Anocentor nitens (Neumann, 1897) é, no Brasil, uma das principais espécies de carrapatos dos equídeos (Borges e Leite, 1993). Tem grande importância na medicina veterinária por ser transmissor da Babesia caballi. Localizado preferencialmente no pavilhão auricular, pode, ainda, em altas infestações, causar lesões deformantes e mutilantes na cartilagem do pavilhão auricular (orelha) (Pfeifer Barbosa, 1993; Bittencourt et al., 1999; Faustino et al., 2005).

O Rhipicephalus sanguineus (Latreille, 1806) é o carrapato natural do cão doméstico, amplamente distribuído no mundo, que pode parasitar diversos hospedeiros, entre eles o homem, devido à sua baixa especificidade (Venzal et al., 2003; Louly et al., 2006; Ribeiro et al., 2006). Ele é um ixodídeo transmissor de vários agentes patogênicos para humanos e animais (Yoshinari et al., 1997; Rozental et al., 2002; Reis et al., 2005).

Vários métodos de controle desses artrópodes são empregados baseados na utilização dos acaricidas químicos. O uso indiscriminado e intenso desses produtos ao longo dos anos tem ocasionado problemas de populações resistentes, além de determinar permanência de resíduos em produtos de origem animal e no meio ambiente (Bittencourt et al., 1999; Labruna, 2004; Farias et al., 2008). Existe uma tendência mundial para reduzir a utilização de acaricidas e inseticidas químicos tanto quanto possível, principalmente devido aos danos causados ao ambiente e à cadeia alimentar. $\mathrm{O}$ uso de plantas com propriedades inseticidas constitui uma alternativa com menor impacto ambiental e representa diminuição de problema de ocorrência de cepas resistentes (Hernández et al., 1987; Fernandes et al., 2007; Valente et al., 2007). A andiroba (Carapa guianensis Aubl.), da família Meliaceae, uma árvore de grande porte encontrada na floresta amazônica, é uma espécie de uso múltiplo cujo óleo, extraído de suas sementes, é bastante utilizado na indústria de cosméticos e na medicina popular da região Norte do Brasil (Loureiro et al., 1979; Hammer e Johns, 1993;
Ferraz et al., 2002; Ficha..., 2005). Contudo não há relatos de sua ação contra ácaros.

O objetivo deste trabalho foi avaliar potencial acaricida in vitro do óleo da semente da andiroba contra A. nitens e $R$. sanguineus.

\section{MATERIAL E MÉTODOS}

Os testes in vitro foram realizados no Laboratório de Doenças Parasitárias dos Animais Domésticos, da área de Medicina Veterinária Preventiva do Departamento de Medicina Veterinária da Universidade Federal Rural de Pernambuco. Para avaliação do potencial acaricida do óleo da semente da andiroba contra amostras de A. nitens $e R$. sanguineus, foi realizado o teste de imersão de fêmeas ingurgitadas, segundo Drummond et al. (1971, 1973), utilizando-se como material botânico um extrato hexânico da semente de andiroba. A extração foi realizada por percolação a frio, com oito litros de solvente polar (98\% etanol comercial) por amostra extraída, e processada na Central de Extração do Departamento de Química, UFPA.

As sementes utilizadas foram coletadas no município de Curuçá, PA, atendendo aos protocolos para obtenção de patentes. As exsicatas foram depositadas no herbário do Museu Paraense Emilio Goeldi, instituição credenciada pelo Instituto Brasileiro do Meio Ambiente e dos Recursos Naturais Renováveis. As coletas foram feitas de acordo com a legislação de acesso ao Patrimônio Genético Nacional (MP N $\mathrm{N}^{0}$ 2.186-16 de 23/08/2001), e foi utilizada a licença de acesso do Convênio UFPA/Extracta Moléculas Naturais S.A.

As fêmeas ingurgitadas de $A$. nitens $(\mathrm{n}=210)$ e $R$. sanguineus $(\mathrm{n}=140)$ foram coletadas manualmente de hospedeiros naturalmente infestados, sendo, respectivamente, equinos do município de Paudalho - mesorregião da zona da mata de Pernambuco - e cães do município de Caruaru - mesorregião do agreste do mesmo Estado - as quais foram transportadas, separadamente por espécies, em recipientes plásticos com aeração adequada. No laboratório, foram limpas com papel absorvente e distribuídas em grupos de 10, sendo a seleção feita com base nos aspectos de aparência e motilidade normais, corpo íntegro e máximo de ingurgitamento (Leite et al., 1995). Os grupos de teleóginas foram transferidos para placas de Petri devidamente identificadas e pesados em balança analítica.

Foram empregadas cinco diluições do óleo de andiroba, $100 \%, \quad 50 \%, \quad 30 \%, 25 \%$ e $10 \%$, 
utilizando-se água destilada e tween 80 como dispersante, em três repetições - por tratamento para o teste com $A$. nitens e duas para $R$. sanguineus, formando-se, ainda, dois gruposcontrole, um apenas com água destilada, e outro com tween 80 e água destilada.

Cada grupo de 10 fêmeas ingurgitadas foi submetido à imersão em copos descartáveis de $50 \mathrm{~mL}$, cada um deles com $10 \mathrm{~mL}$ das soluções testadas, mantendo-se em constante agitação durante cinco minutos, após os quais o líquido foi desprezado e o excesso das soluções retirado com papel absorvente. Os grupos testados foram recolocados na placa de Petri e mantidos no laboratório em temperatura ambiente, com umidade relativa e temperatura aferidas diariamente por um termo-higrômetro digital, modelo Termohygro - TFA, instalado no local. Transcorridos 10 dias do início da postura, a massa de ovos de cada grupo foi pesada em balança analítica, transferida para seringas plásticas descartáveis de $20 \mathrm{~mL}$ previamente adaptadas, identificadas e vedadas com algodão hidrófobo, mantendo-se as mesmas condições anteriormente descritas. Após o período de incubação, o percentual de eclosão foi estimado objetivamente, estabelecendo-se como parâmetro a verificação visual com intervalos de $10 \%$.

O potencial acaricida foi estimado por cálculo da eficácia do produto (EP), segundo Drummond et al. (1973). De acordo com a legislação pertinente à comercialização de carrapaticidas no país (Portaria..., 1990), os resultados foram interpretados considerando-se como eficácia o valor mínimo de $95 \%$.

\section{RESULTADOS E DISCUSSÃO}

Os resultados obtidos para verificar o potencial acaricida in vitro do óleo da semente da andiroba sobre $A$. nitens e $R$. sanguineus demonstraram $100 \%$ de eficácia para todas as diluições testadas (Tab. 1 e 2).

Tabela 1. Atividade do óleo da semente de andiroba (Carapa guianensis Aubl.) sobre fêmeas ingurgitadas de Anocentor nitens

\begin{tabular}{ccccc}
\hline Tratamento & $\begin{array}{c}\text { Peso da teleógina } \\
(\mathrm{g})\end{array}$ & $\begin{array}{c}\text { Peso dos } \\
\text { ovos }(\mathrm{g})\end{array}$ & $\begin{array}{c}\text { Eclosão } \\
(\%)\end{array}$ & $\begin{array}{c}\text { Eficácia do } \\
\text { produto }(\%)\end{array}$ \\
\hline T1 & 2,3549 & 0 & 0 & 100 \\
T2 & 2,3552 & 0 & 0 & 100 \\
T3 & 2,3562 & 0 & 0 & 100 \\
T4 & 2,3537 & 0 & 0 & 100 \\
T5 & 2,3589 & 0,02375 & 0 & 100 \\
C1 & 2,3515 & 1,1168 & 100 & - \\
C2 & 2,3523 & 0,9845 & 100 & - \\
\hline
\end{tabular}

T1: 100\% óleo de andiroba; T2: 50\% óleo de andiroba; T3: 30\% óleo de andiroba; T4: $25 \%$ óleo de andiroba; T5: $10 \%$ óleo de andiroba; $\mathrm{C} 1$ : controle de água destilada; $\mathrm{C} 2$ : controle de água destilada + tween 80.

Tabela 2. Atividade do óleo da semente de andiroba (Carapa guianensis Aubl.) sobre fêmeas ingurgitadas de Rhipicephalus sanguineus

\begin{tabular}{ccccc}
\hline Tratamento & $\begin{array}{c}\text { Peso da teleógina } \\
(\mathrm{g})\end{array}$ & $\begin{array}{c}\text { Peso dos } \\
\text { ovos }(\mathrm{g})\end{array}$ & $\begin{array}{c}\text { Eclosão } \\
(\%)\end{array}$ & $\begin{array}{c}\text { Eficácia do } \\
\text { produto }(\%)\end{array}$ \\
\hline T1 & 0,98185 & 0 & 0 & 100 \\
T2 & 0,98525 & 0 & 0 & 100 \\
T3 & 0,95245 & 0 & 0 & 100 \\
T4 & 0,9534 & 0 & 0 & 100 \\
T5 & 0,9555 & 0,02249 & 0 & 100 \\
C1 & 0,99655 & 0,6936 & 100 & - \\
C2 & 0,9957 & 0,6722 & 100 & - \\
\hline
\end{tabular}

T1: 100\% óleo de andiroba; T2: 50\% óleo de andiroba; T3: $30 \%$ óleo de andiroba; T4: $25 \%$ óleo de andiroba; T5: $10 \%$ óleo de andiroba; $\mathrm{C} 1$ : controle de água destilada; $\mathrm{C} 2$ : controle de água destilada + tween 80. 
Em ambas as espécies de carrapatos, observou-se o percentual de $100 \%$ sobre a mortalidade da fêmea ingurgitada em todas as diluições, entre o terceiro e o quarto dia após o teste, exceto na diluição a $10 \%$, cuja mortalidade foi de $90 \%$ no segundo dia após tratamento. No sexto dia após o teste, observou-se $100 \%$ de mortalidade. Nesta diluição, verificou-se postura de ovos inférteis. Em todos os grupos-controle também ocorreu postura que se iniciou no segundo dia após o tratamento, com $100 \%$ de eclosão. As médias referentes à temperatura e umidade relativa registradas no laboratório durante o período experimental foram $29^{\circ} \mathrm{C}$ e $75 \%$, respectivamente, condições essas adequadas para as teleóginas efetuarem postura e ocorrer eclosão de larvas, de acordo com Faustino et al. (2005) e Amaro et al. (2006).

Alguns estudos utilizaram o óleo da andiroba como fitoterápico Miot et al. (2004) concluíram que o óleo puro de andiroba apresenta efeito repelente discreto contra picada de Aedes sp., o que é significativamente inferior ao DEET 50\% (N,N-diethyl-M-toluamide). Silva et al. (2004), Emerick et al. (2005) e Rossi et al. (2005), em larvas de terceiro e quarto estágios de Aedes albopictus, mosquitos do gênero Culex e Aedes aegypti, respectivamente, comprovaram a ação larvicida em diferentes diluições do óleo de andiroba. Oliveira et al. (2006) observaram atividade inseticida do óleo bruto da andiroba sobre operárias de Atta sexdens rubropilosa (Hymenoptera: Formicidae).

Os resultados apresentados são semelhantes aos obtidos por Sousa et al. (2005) que, ao utilizarem as diluições de $50 \%$ e $100 \%$ sobre $A$. nitens, obtiveram $100 \%$ de eficácia, e por Farias et al. (2007), que, com as mesmas diluições do óleo de andiroba sobre B. microplus, obtiveram $100 \%$ de mortalidade das fêmeas ingurgitadas com inibição da ovipostura.

\section{CONCLUSÃO}

A atividade biológica in vitro do óleo da semente da andiroba sobre fêmeas ingurgitadas de $A$. nitens e $R$. sanguineus apresenta potencialidade acaricida sobre essas espécies de ixodídeos.

\section{REFERÊNCIAS BIBLIOGRÁFICAS}

AMARO, N.B.; D'ALESSANDRO, W.B.; CASTRO JÚNIOR, L.C. et al. Estudo da atividade larvicida de Trichilia swartz (Meliaceae) para o controle de Rhipicephalus sanguineus (Latreille, 1806) (Acari: Ixodidae). In: CONGRESSO BRASILEIRO DE ENTOMOLOGIA, 21., 2006, Recife. Anais... Recife, 2006. CD-ROM.

BITTENCOURT, V.R.E.P.; MENEZES, G.C.R.; MASCARENHAS, A.G. et al. Ação dos fungos Beauveria bassiana (Balsamo) Vuillemin, 1912 e Metarhizium anisopliae (Metschnikoff, 1879) Sorokin, 1883 sobre larvas do carrapato Anocentor nitens (Acari: Ixodidae). Parasitol. Dia, v.23, p.82-86, 1999.

BORGES, L.M.F.; LEITE, R.C. Comparação entre as populações auriculares e nasais de Dermacentor nitens (Neumann, 1897) oriundas de equinos de Minas Gerais e Bahia. Rev. Bras. Parasitol. Vet., v.2, p.109-110, 1993.

DENNING, F. Unsucessfull attemps to transmit Amblyomma cajennense. 1988. 112f. Thesis Tierärztliche Hochschule Hannover (TIHO), Hannover.

DRUMMOND, R.O.; ERNST, S.E.; TREVINO, J.L. et al. Boophilus annulatus and Boophilus microplus: Laboratory tests of insecticides. J. Econ. Entomol., v.66, p.130-133, 1973.

DRUMMOND, R.O.; GLADNEY, W.J.; WHETSTONE, T.M. et al. Laboratory tests of insecticides for control of the winter tick. $J$. Econ. Entomol., v.64, p.686-688, 1971.

EMERICK, S.; PROPHIRO, J.; ROSSI, J. et al. Resultados preliminares do efeito larvicida do óleo de andiroba (Carapa guianensis) (Meliacea) em mosquitos do gênero Culex (Diptera: Culicidae). In: CONGRESSO BRASILEIRO de MEDICINA TROPICAL, 41., 2005, Florianópolis. Anais...Florianópolis, [s.n.], 2005. p.44-45. (Resumo).

FARIAS, M.P.O; SOUSA, D.P.; ARRUDA, A.C. et al. Eficácia "in vitro"do óleo da Carapa guianensis AUBL. (Andiroba) no controle do Boophilus microplus (Canestrini, 1887) (Acari: Ixodidae). Rev. Bras. Plantas Medic., v.9, p.68$71,2007$. 
FARIAS, N.A.; RUAS, J.L.; SANTOS, T.R.B. Análise da eficácia de acaricidas sobre Boophilus microplus, durante a última década, na região do Rio Grande do Sul. Cienc. Rural, v.38, p.17001704, 2008.

FAUSTINO, M.A.G.; RAMOS, J.V.A.; OLIVEIRA, M.P.B. et al. Estudo comparativo de dados bioecológicos da fase não parasitária de Anocentor nitens (Acari: Ixodidae) (Neumann, 1897) em dois ambientes experimentais no Recife-PE. Cienc. Vet. Trop., v.8, p.43-52, 2005.

FERNANDES, F.F.; LELES, R.N.; SILVA, I.G. et al. Larvicidal potencial of Sapindus saponaria (Sapindaceae) against Rhipicephalus sanguineus (Latreille, 1806) (Acari: Ixodidae). Arq. Bras. Med. Vet. Zootec., v.59, p.145-149, 2007.

FERRAZ, I.D.K.; CAMARGO, J.L.C.; SAMPAIO, P.T.B. Sementes e plântulas de andiroba (Carapa guianensis AUBL. e Carapa procera D.C.): aspectos botânicos, ecológicos e tecnológicos. Acta Amaz., v.32, p.647-661, 2002.

FICHA da planta Andiroba. [s.1.: s.n.], 2005 Disponível em: http://globorural.globo.com/barra.asp?d=/edic/16 9/fichaplanta1.htm. Acessado em: 31 jan. 2005.

HAMMER, M.L.A.; JOHNS, E.A. Tapping an Amazonian plethora: four medicinal plants of Marajá Islands, Pará (Brazil). J. Ethnophamacol., v.40, p.53-75, 1993.

HERNÁNDEZ, L.E.; PARRA, D.G.; MARIN, A.C. Accion repelente y acaricida del Melinis minutiflora sobre el Boophilus microplus . Rev. Colomb. Cienc. Quim. Farm., v.16, p.17-21, 1987.

LABRUNA, M.B. Biologia-ecologia de Rhipicephalus sanguineus (Acari:Ixodidae). Rev. Bras. Parasitol. Vet., v.13, supl., p.123-124, 2004.

LABRUNA, M.B.; PEREIRA, M.C. Febre maculosa: aspectos clínico-epidemiológicos. Clin. Vet., v.12, p.19-23, 2001.

LEITE, R.C.; LABRUNA, M.B.; OLIVEIRA, P.R. et al. In vitro susceptibility of engorged females from different populations of Boophilus microplus to comercial acaricide. Rev. Bras. Parasitol. Vet., v.4, p.283-294, 1995.

LOULY, C.C.B.; FONSECA, I.N.; OLIVEIRA, V.F. et al. Ocorrência de Rhipicephalus sanguineus em trabalhadores de clínicas veterinárias e canis, no município de Goiânia, GO. Cienc. Anim. Bras., v.7, p.103-106, 2006.

LOUREIRO, A.A.; SILVA, M.F.; ALENCAR, J.C. Essências madeireiras da Amazônia. Manaus: INPA/Suframa, 1979. v.1, 245p.

MIOT, H.A.; BATISTELLA, R.F.; BATISTA, K.A. et al. Comparative study of the topical effectiveness of the andiroba oil (Carapa guianensis) and DEET $50 \%$ as repellent for Aedes sp. Rev. Inst. Med. Trop. S. Paulo, v.45, p.253-256, 2004.

MONTEIRO, S.G.; BAHIENSE, T.C.; BITTENCOURT, V.R.E.P. Ação do fungo Beauveria bassian (Balsamo) Vuillemin, 1912, sobre a fase parasitária do carrapato Anocentor nitens (Neumann, 1897) Schulse, 1937 (Acari: ixodidae). Cienc. Rural, v.33, p.559-563, 2003.

OLIVEIRA, M.F.S., REISS, I.C.; BUENO, F.C. et al. Toxicidade do óleo bruto de andiroba (Carapa guianensis) para operárias de Atta sexdens rubropilosa (Hymenoptera: Furmicidae). In: CONGRESSO BRASILEIRO DE ENTOMOLOGIA, 21., 2006, Recife. Anais... Recife, 2006. CD-ROM.

PFEIFER BARBOSA, I.D. Epidemiological studies of infections with Babesia equi and Babesia caballi in Brazil. 1993. 62f. Dissertation (Doctorat) - Tierärztliche Hochschule Hannover (TIHO), Hannover.

PORTARIA n.90 de 04 de dez. de 1989. Normas para produção, controle e utilização de produtos antiparasitários [do Ministério da Agricultura]. Diário Oficial, 22 jan. 1990. sec.1, col.2.

REIS, R.C.S.; MELO, D.R.; PERINOTTO, W.M.S. et al. Patogenicidade in vitro de formulações fúngicas sobre ninfas e adultos de Rhipicephalus sanguineus (Latreile, 1806) (Acari: Ixodidae). Rev. Bras. Parasitol. Vet., v.14, p.101-105, 2005.

RIBEIRO, F.A.; FERNANDES, J.I.; CORREIA, T.R. et al. Eficácia "in vitro" de diferentes acaricidas no controle de fêmeas ingurgitadas de Rhipicephalus sanguineus. In: CONGRESSO BRASILEIRO DE PARASITOLOGIA VETERINÁRIA E SIMPÓSIO LATINOAMERICANO DE RIQUETSIOSES, 14., 2006, Ribeirão Preto. Anais..... Ribeirão Preto: [s.n.], 2006. p.225. (Resumo). 
ROSSI, J.N.C.; PROPHIRO, J.S.; PEDROSO, M.F. et al. Uso do óleo de andiroba (Carapa guianensis - Meliaceae) como larvicida de Aedes aegypti (Diptera: Culicidae). In: CONGRESSO DA SOCIEDADE BRASILEIRA DE MEDICINA TROPICAL, 41., 2005, Florianópolis. Anais... Florianóplis, [s.n.], 2005. p.78. (Resumo).

ROZENTAL, T.; BUSTAMANTE, M.C.; AMORIM, M. et al. Evidence of spotted fever group rickettsae in state of Rio de Janeiro, Brazil. Rev. Inst. Med. Trop. S. Paulo, v.44, p.155-158, 2002.

SILVA, O.S.; ROMÃO, P.R.T.; BLAZIUS, R.D. et al. The use of andiroba Carapa guianensis as larvicide against Aedes albopictus. J. Am. Mosq. Control Assoc., v.20, p.456-457, 2004.

SOARES, S.F.E; BORGES, L.M.F.; FERRI, P.H. et al. Avaliação da eficácia de Melia azedarach (Meliacea) sobre Rhipicephalus sanguineus. In: CONGRESSO BRASILEIRO DE PARASITOLOGIA VETERINÁRIA; SIMPÓSIO LATINO-AMERICANO DE
RIQUETSIOSES, 14., Ribeirão Preto. Anais..... Ribeirão Preto, [s.n.], 2006. p.184. (Resumo).

SOUSA, D.P.; OLIVEIRA, M.P.B.; FAUSTINO, M.A.G. et al. Avaliação "in vitro" da atividade do extrato de andiroba (Carapa guianensis) sobre fêmeas ingurgitadas de Anocentor nitens (Acari: Ixodidae). In: JORNADA DE ENSINO, PESQUISA E EXTENSÃO, 5., Recife. Anais...Recife: UFRPE, 2005. CD- ROM.

VALENTE, M.; BARRANCO, A.; SELLAIVEVILLAROEL, A.B. Eficácia do extrato aquoso de Azadiracta indica no controle de Boophilus microplus em bovino. Arq. Bras. Med. Vet. Zootec., v.59, p.1341-1343, 2007.

VENZAL, J.M.; GUGLIELMONE, A.A.; ESTRADA-PENA, A. et al. Ticks (Ixodida: Ixodidae) parasitising humans in Uruguay. Trop. Med. Parasitol., v.97, p.769-772, 2003.

YOSHINARI, N.H.; BARROS, P.J.L.; BONOLDI, V.L.N. et al. Perfil da borreliose de Lyme no Brasil. Rev. Hosp. Clin., v.52, p.111$117,1997$. 